\title{
Influence of the Black Hole Rotation on Accretion Discs and Jets in Active Galaxies: the Case of NGC 1068
}

\author{
Anderson Caproni, Zulema Abraham, \\ Instituto de Astronomia, Geofísica e Ciências Atmosféricas, Universidade de São Paulo, \\ R. do Matão 1226, Cidade Universitária, CEP 05508-900, São Paulo, SP, Brazil; \\ and Herman J. Mosquera Cuesta* \\ Instituto de Cosmologia, Relatividade e Astrofísica (ICRA-BR), Centro Brasileiro de Pesquisas Físicas, \\ R. Dr. Xavier Sigaud 150, CEP 22290-180, Rio de Janeiro, RJ, Brazil
}

(Received on 14 October, 2005)

\begin{abstract}
Although we do not observe directly black holes in the Universe, their presence has been inferred in several astrophysical systems, from galactic to extragalactic scales. In this work, we will restrict our focus in the possible signatures of the presence of Kerr black holes in active galactic nuclei, one of the most powerful sources in the Universe. Particularly, we will discuss how jet/accretion disc precession and their directional stability can be used to trace the accretion disc properties as well as the black hole spin in the Seyfert galaxy NGC 1068.
\end{abstract}

\section{INTRODUCTION}

A fraction of galaxies in the Universe exhibit an unusual release of energy from their central regions; such objects are known as active galactic nuclei (AGNs). This class contains quasars, BL Lac objects and Seyfert galaxies and is basically characterized by high luminosity $\left(10^{35}-10^{41} \mathrm{~W}\right)$ from radio to $\mathrm{X}$-rays wavelengths (some cases to $\mathrm{TeV}$ energies), produced in a small volume $\left(<1 \mathrm{pc}^{3}\right)$. Their spectra show the presence of several optical emission lines associated to several chemical elements, which contrasts to the normal galaxies, detected predominantly in absorption. Variability of the continuum and/or the emission lines are often observed in timescales from days to years.

It is a well-accepted idea in the astrophysical community that the center of an AGN harbours a supermassive black hole surrounded by an accretion disc. The accretion process of the disc material onto the black hole is believed to be the main source of the copious energy production, even though the complete understanding of this process has not been achieved up to now; several theoretical models have been proposed to explain the characteristics of the accretion mechanism (e.g., Ref. 1). Numerical simulations also have been carried on in order to tackle the non-linearity of the differential equations that rule the accretion disc (e.g., Ref. 2).

Not rarely, there is ejection of bipolar collimated structures from the innermost part of the AGN known as jets. Some jets are accelerated to high speeds, exceeding ninety percent of the light speed in many cases [3].

Some AGNs exhibit distortions in the jet morphology, such as changes in its direction of propagation on the plane of the sky (e.g., Ref. 4), as well as in the apparent proper motion (e.g., Ref. 5). Those odd aspects can be generated by jet precession, since it introduces a periodic time variation of the

*Also at Abdus Salam International Centre for Theoretical Physics, Strada Costiera 11, Miramare 34014, Trieste, Italy. jet viewing angle in relation to the line-of-sight [4, 6-10]. In the case of Seyfert galaxies, precession was detected from the variability of double-peaked Balmer lines produced in the accretion disc (e.g., Ref. 11).

The existence of a warped accretion disc in the Seyfert 2 galaxy NGC 1068 has been inferred from the kinematics of the maser spots at parsec-scales from very long baseline radio interferometry (e.g., Ref. 12). Warping and disc/jet precession can be produced in a supermassive black hole binary system wherein the primary accretion disc precesses because of torques induced by the secondary black hole, whose orbital plane does not coincide with that of the accretion disc $[9,10,13,14]$.

In this work, we will focus our attention on another feasible scenario to produce warp and precession of the disc: the Bardeen-Petterson effect [15]. A general introduction about this mechanism will be presented in Section II. A brief description about the Seyfert 2 galaxy NGC 1068, as well as the application of the Bardeen-Petterson formalism to this source is made in Section III. Conclusions are presented in Section IV.

\section{THE BARDEEN-PETTERSON EFFECT}

Frame dragging produced by a Kerr black hole causes precession of a particle if its orbital plane is inclined in relation to the equatorial plane of the black hole. The precession angular velocity $\Omega_{\mathrm{LT}}$ due to the Lense-Thirring effect is given by [16]:

$$
\Omega_{\mathrm{LT}}=\frac{2 G}{c^{2}} \frac{J_{\mathrm{BH}}}{r^{3}},
$$

where $G$ is the gravitational constant, $c$ is the light speed, $r$ is the radial distance from the black hole and $J_{\mathrm{BH}}$ is the angular momentum of the black hole, defined as $J_{\mathrm{BH}}=a_{*} G M_{\mathrm{BH}}^{2} / c$. The parameters $M_{\mathrm{BH}}$ and $a_{*}$ correspond respectively to the black hole mass and the ratio of the angular momentum of the compact object and that of a Kerr black hole rotating at 


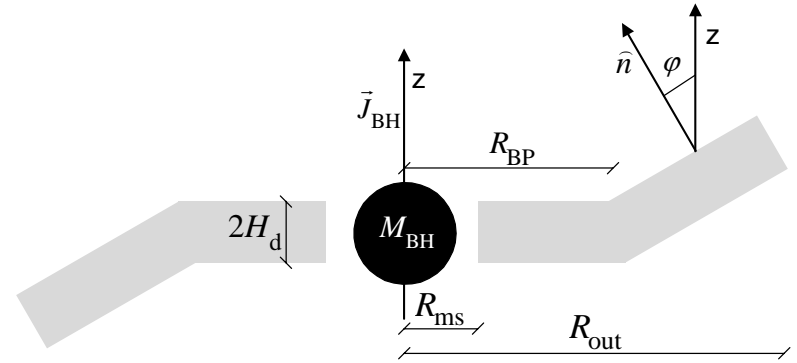

FIG. 1: Schematic representation of the Bardeen-Petterson effect. An accretion disc with inner and outer radius $R_{\mathrm{ms}}$ and $R_{\text {out }}$ respectively, having a semi-thickness $H_{\mathrm{d}}$ and misaligned initially by an angle $\varphi$ in relation to the angular momentum of The black hole $J_{\mathrm{BH}}$ and mass $M_{\mathrm{BH}}$, will be warped by the Bardeen-Petterson effect. The transition radius between the portion of the disc that is aligned and misaligned with the black hole's equator is known as the BardeenPetterson radius $R_{\mathrm{BP}}$.

its maximal velocity. The spin-induced precession formalism has been successfully applied to supermassive black holes in AGNs and SgrA*, the centre of our Galaxy [17].

The combined action of the Lense-Thirring effect and the internal viscosity of the accretion disc forces the alignment between the angular momenta of the Kerr black hole and the accretion disc. This is known as the Bardeen-Petterson ef- fect [15] and usually affects only the innermost part of the disc, while its outer parts tend to remain in its original configuration, due to the short range of the Lense-Thirring effect. The transition radius between these two regimes is known as Bardeen-Petterson radius $R_{\mathrm{BP}}$, shown schematically in Fig. 1, and its exact location depends mainly on the physical properties of the accretion disc [18-22].

A rough estimate of $R_{\mathrm{BP}}$ can be obtained comparing the time-scales for Lense-Thirring precession and warp transmission through the disc [23] that, on the other hand, will depend on how the warps are being communicated along it. If they are transmitted diffusively $R_{\mathrm{BP}}^{\text {dif }}=\sqrt{v_{2}\left(R_{\mathrm{BP}}^{\text {dif }}\right) / \Omega_{\mathrm{LT}}\left(R_{\mathrm{BP}}^{\text {dif }}\right)}$, where $v_{2}$ is the viscosity acting on the perpendicular direction to the disc, related to the disc viscosity $v_{1}$ measured in the horizontal plane. If the time-scale for the warp evolution is similar to that for the evolution of the surface density, $v_{1} \sim v_{2}$; otherwise $v_{2} \sim f(\alpha) v_{1}$, where $f(\alpha)$ is a function of the dimensionless viscosity parameter $\alpha$ introduced by [1]. For typical parameters of AGNs, it is expected $v_{2} \gg v_{1}$ [24], so that the Bardeen-Petterson radius is substantially reduced in this case. In this work, we will follow [25], adopting $f(\alpha)=2\left(1+7 \alpha^{2}\right) /\left[\alpha\left(4+\alpha^{2}\right)\right]$.

Assuming the zero torque inner boundary condition at the marginally stable orbit $R_{\mathrm{ms}}$, considered hereafter the inner radius of the accretion disc, the viscosity $v_{1}$ can be written as:

$$
v_{1}=-\frac{\dot{M}}{2 \pi \Sigma_{\mathrm{d}}(r)}\left[\frac{d \ln \Omega_{\mathrm{d}}(r)}{d \ln r}\right]^{-1}\left[1-\left(\frac{R_{\mathrm{ms}}}{r}\right)^{2} \frac{\Omega_{\mathrm{d}}\left(R_{\mathrm{ms}}\right)}{\Omega_{\mathrm{d}}(r)}\right]
$$

where $\dot{M}$ is the accretion rate, $\Sigma_{\mathrm{d}}$ is the surface density of the accretion disc integrated over the semi-thickness of the disc $H_{\mathrm{d}}$ and $\Omega_{\mathrm{d}}$ is the angular velocity of the disc.

On the other hand, the Bardeen-Petterson radius can be estimated in the wave-like regime using $R_{\mathrm{BP}}^{\mathrm{W}}=$ $c_{\mathrm{s}}\left(R_{\mathrm{BP}}^{\mathrm{W}}\right) / \Omega_{\mathrm{LT}}\left(R_{\mathrm{BP}}^{\mathrm{W}}\right)$, where $c_{\mathrm{s}}$ is the sound-cross speed of the disc, defined as:

$$
c_{\mathrm{s}}(r)=\sqrt{-\Gamma \frac{d \ln \Omega_{\mathrm{d}}(r)}{d \ln r} \frac{v_{1}(r) \Omega_{\mathrm{d}}(r)}{\alpha}},
$$

where $\Gamma$ is the politropic index of the equation-of-state of the gas (we have assumed $\Gamma=5 / 3$ in this work). The transition from the diffusive to wave-like regime occurs at a radius $R_{\mathrm{T}}$ where $\alpha \sim H_{\mathrm{d}} / r$ [26].

The time-scale for the black hole to align its angular momentum with that of the accretion disc was firstly estimated by [27], assuming that each mass element accreted by the black hole carries an orbital angular momentum corresponding to that found at the Bardeen-Petterson radius. [28] obtained an analytic solution to the equations that control the warp evolution in the case of a disc with constant surface density and calculated the alignment time-scale. [23] generalizes the results found by [28] to a power-law viscosity. Such studies suggest that the alignment time-scale can be estimated using:

$$
T_{\text {align }}=J_{\mathrm{BH}}\left(\frac{d J_{\mathrm{BH}}}{d t}\right)^{-1} \sin \varphi
$$

where $\varphi$ is the inclination angle between the normal of the outer parts of the disc and the spin axis of the black hole. The time derivative of $J_{\mathrm{BH}}$ has the form [23]:

$$
\frac{d J_{\mathrm{BH}}}{d t}=-2 \pi \sin \varphi{ }_{R_{\mathrm{BP}}}^{\mathrm{Z}} \Omega_{\mathrm{out}}(r) L_{\mathrm{d}}(r) r d r
$$

where $R_{\text {out }}$ is the outer radius of the disc and $L_{\mathrm{d}}(r)=$ $\Sigma_{\mathrm{d}}(r) \Omega_{\mathrm{d}}(r) r^{2}$ is its angular momentum density (e.g., Ref. 17). 


\section{BARDEEN-PETTERSON EFFECT: WARPING AND PRECESSING THE ACCRETION DISC OF NGC 1068}

In this section, we will discuss how to constrain the physical properties of the accretion disc and/or the black hole spin in the case of a Bardeen-Petterson disc, using NGC 1068 as example.

\section{A. NGC 1068 and its accretion disc parameters}

The Seyfert 2 galaxy NGC 1068 is located at a distance of 14.4 Mpc [29]. Its central engine is obscured by a dust torus, which was recently spatially resolved by mid-infrared interferometric observations [30], in which it was detected a distribution of warm dust in a parsec-scale structure, surrounding a smaller hot source (probably the accretion disc).

Very long baseline interferometry of NGC 1068 at radio wavelengths reveals a complex jet-like structure, essentially composed by four discrete components at sub-arc-seconds scale labeled as S2, S1, C and NE (e.g., Ref. 12). The component $\mathrm{S} 1$ is probably associated with the thin accretion disc that surrounds a black hole while the other structures are possibly related to jet components (e.g., Ref. 12). The radio jet orientation change with the core distance, bending towards the northeast direction, specially at component C. At larger scales, extended lobes are observed, with the northeast lobe having a conical shape, similar to a bow shock structure produced by jet-environment interaction [31].

The nuclear region of NGC 1068 has been also investigated through $\mathrm{H}_{2} \mathrm{O}$ and $\mathrm{OH}$ maser emission (Refs. 12, 32), with the brightest $\mathrm{H}_{2} \mathrm{O}$ maser spots coinciding with the radio continuum component $\mathrm{S} 1$ [33]. The maser emission seems to originate from a sub-Keplerian rotating disc with inner and outer radius corresponding to $\sim 0.65$ and $1.1 \mathrm{pc}$ respectively, enclosing a mass of $\sim 10^{7} \mathrm{M}_{\odot}$ within its inner radius [32, 34]. The departure from Keplerian motions has been attributed to a massive accretion disc that dilutes the gravitational field of the black hole $[35,36]$.

A very interesting result from maser observations is that the maser disc is not completely aligned with the major axis of the component S1 (e.g., Ref. 12). Indeed, they reveal a maser distribution similar to a warped disc, with the inner parts almost perpendicular to the jet and aligned to the major axis of S1, but deviating from this configuration as the core distance increases. Recently, [12] proposed a configuration in which the thin hot disc (S1) is perpendicular to the radio jet, while the misaligned maser disc points towards the parsec-scale dustytorus.

\section{B. Bardeen-Petterson radius and alignment time-scale}

From the analysis of the rotation curve of the maser emission in NGC 1068, [35] and [36] determined a mass of about $1.2 \times 10^{7}$ and $8 \times 10^{6} \mathrm{M}_{\odot}$ respectively for the supermassive black hole. [12] found a lower limit of $\sim 7 \times 10^{37} \mathrm{~W}$ for the
TABLE I: Physical parameters of the accretion disc of the Seyfert 2 galaxy NGC 1068.

\begin{tabular}{lcc}
\hline \hline Parameter & Model A & Model B \\
\hline$M_{\mathrm{BH}}\left(\mathrm{M}_{\odot}\right)$ & $(1.2 \pm 0.1) \times 10^{7}$ & $(8.0 \pm 0.3) \times 10^{6}$ \\
$s$ & $-1.05 \pm 0.10$ & $-1.05 \pm 0.10$ \\
$\Sigma_{0}\left(\mathrm{~kg} \mathrm{~m}^{-2}\right)$ & $(1.06 \pm 0.23) \times 10^{10}$ & $(1.49 \pm 0.18) \times 10^{10}$ \\
$\dot{M} / \dot{M}_{\text {Edd }}$ & $0.46-1.0$ & $0.71-1.0$ \\
$\alpha \varepsilon\left(a_{*}\right)$ & $4.4 \times 10^{-4}-9.4 \times 10^{-4}$ & $4.4 \times 10^{-4}-6.3 \times 10^{-4}$ \\
\hline \hline
\end{tabular}

bolometric luminosity $L_{\text {bol }}$ of NGC 1068 to reproduce the observed free-free emission. We will consider that such bolometric luminosity extends from this value to the Eddington luminosity.

We also assumed a power-law surface density for the accretion disc (integrated over the scale height of the accretion disc) $\Sigma(r)=\Sigma_{0}\left(r / R_{\mathrm{g}}\right)^{s}$, with $s=-1.05$ [35] and $R_{\mathrm{g}}=G M_{\mathrm{BH}} / c^{2}$, the gravitational radius. The constant $\Sigma_{0}$ was determined from the mass of the disc $M_{\mathrm{d}}$, integrating $\Sigma(r)$ from the inner radius to the outer radius of the disc.

Limits to the $\alpha$-parameter (multiplied by the accretion efficiency $\varepsilon\left(a_{*}\right)$ ) were found combining Eq. (8) of [36] with the values of bolometric luminosity, which is displayed together other disc parameters in Table 1 . In our analysis, we have considered two different models, labelled as A and B (see Table 1 ), referring respectively to the parameters derived from [35] and [36].

The maser velocities present signatures of sub-Keplerian motion; however, at the radius in which it occurs the disc angular momentum becomes negligible, such that in Eq. (3) we used the relativistic Keplerian angular velocity $\Omega_{\mathrm{K}}$ :

$$
\Omega_{\mathrm{K}}(r)=\frac{c^{3}}{G M_{\mathrm{BH}}}\left[\left(\frac{r}{R_{\mathrm{g}}}\right)^{3 / 2}+a_{*}\right]^{-1} .
$$

In order to estimate the Bardeen-Petterson radius, we should first know where is located the transition radius between the diffusive and wave regimes that, as seen in previous section, will depend on the aspect ratio $H_{\mathrm{d}} / r$. This quantity was derived following [37]:

$$
\frac{H_{\mathrm{d}}(r)}{r}=\frac{1}{r} \frac{H_{\mathrm{nsg}}(r) H_{\mathrm{sg}}(r)}{\sqrt{H_{\mathrm{nsg}}^{2}(r)+H_{\mathrm{sg}}^{2}(r)}}
$$

where $H_{\mathrm{nsg}}=c_{\mathrm{s}} / \Omega_{\mathrm{d}}$ and $H_{\mathrm{sg}}=c_{\mathrm{s}}^{2} /\left(\pi G \Sigma_{\mathrm{d}}\right)$.

We performed calculations of the Bardeen-Petterson radius for the disc models A and B listed in Table 1, considering several values of the black hole spin. The results show that the Bardeen-Petterson radius for NGC 1068 depends weakly on the accretion rate, being limited by the variation of the black hole spin and $\alpha$ parameters; typically, the Bardeen-Petterson radius ranges from $10^{-5}$ to $10^{-4} \mathrm{pc}$ (about 20 and $200 \mathrm{Rg}$ respectively), as shown in left panel of Figure 2.

Except for $a_{*}=0.25$ and 0.5 in the case of highest $\alpha$, the Bardeen-Petterson radius from model A is systematically 

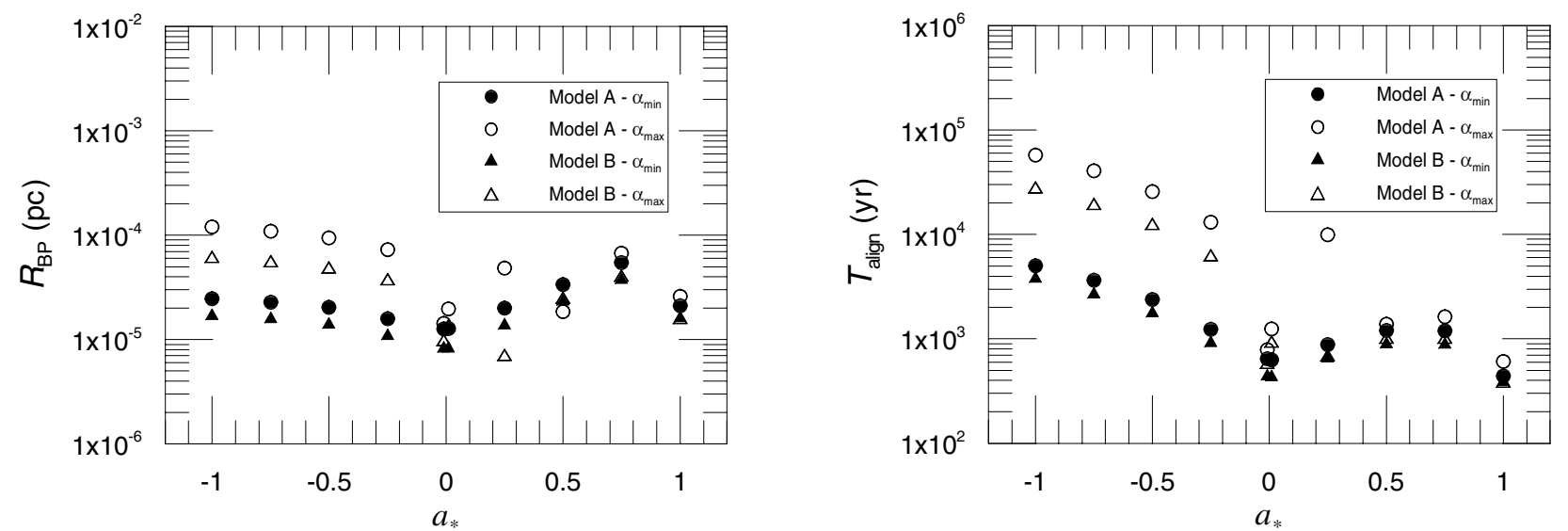

FIG. 2: Left panel: Bardeen-Petterson radius as a function of the black hole spin for NGC 1068. Each point corresponds to the mean value of $R_{\mathrm{BP}}$ considering the limits of minimum and maximum accretion rate. Circles and triangles refer respectively to the models A and B. Filled symbols are related to the lower value of $\alpha$-parameter, while open ones to its upper value. Right panel: Time-scale for alignment between accretion disk and the black hole's equator, using the same nomenclature for the symbols.

larger than that from model B. It can be interpreted as a consequence of the dependency of the Lense-Thirring effect with the squared black hole mass, which is larger for model A. Furthermore, fixing a particular model and a black hole spin, we note that the larger the $\alpha$-parameter is the larger the BardeenPetterson radius is, which is induced by the more efficient coupling between consecutive disc annuli due to increase of disc viscosity. Another aspect seen in Figure 2 is a bump-like feature around $a_{*}=0.75$. It is the net result of the decrease of the Lense-Thirring effect and the increase of the inner radius of the accretion disc with the decrement of the black hole spin.

We calculated the alignment time-scale as a function of the black hole spin by solving the integral given in Eq. (5), using as integration limits the Bardeen-Petterson radius and an arbitrary outer radius. The results, which are independent of the outer radius value, due to the rapid decrease of the angular momentum density of the disk, are presented in the right panel of the Figure 2.

Our estimates of the alignment time-scale are ranged approximately from 100 to $10^{5} \mathrm{yr}$, shorter than the lifetime of the kilo-parsec radio jet of NGC $1068\left(\leq 1.5 \times 10^{5} \mathrm{yr}\right.$; Ref. 38), which must not be so different of the lifetime of the AGN activity. Therefore, our results indicate that the BardeenPetterson effect can perfectly warp the inner part of the accretion disc within its AGN lifetime.

An additional constraint for the alignment time-scale in NGC 1068 can be obtained from its jet morphology. If the accretion disc is forced to align completely with the black hole, we should observe a progressive change in the jet direction. Indeed, there is a clear variation in the position angle among discrete features along the jet (e.g., Ref. 12). In fact, an exponential time variation for the inclination angle between black hole spin and the jet direction, as well as for the precession period $P_{\text {prec }}$ is claimed by [28]:

$$
\varphi(t)=\varphi_{0} e^{-\left(t-t_{0}\right) / T_{\text {align }}}
$$

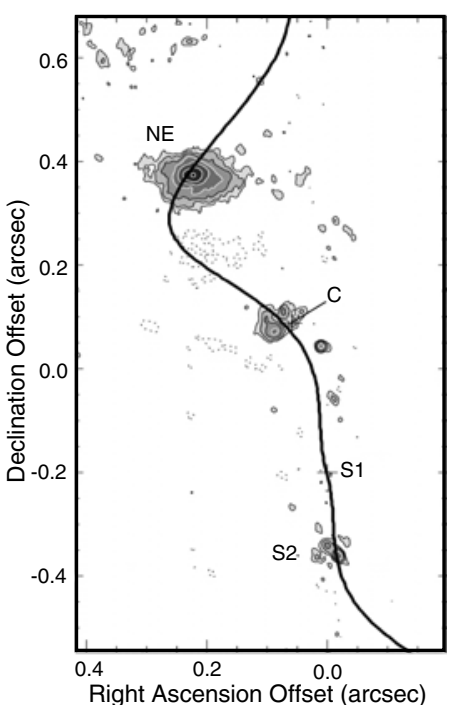

FIG. 3: Snapshot of the variation in the jet orientation due to the Bardeen-Petterson effect. Parsec-scale jet of NGC 1068 at $1.4 \mathrm{GHz}$ [12], represented by the contour map seen in the figure. Solid line indicates the model prediction from Eq. (8) considering $\theta=80 \mathrm{deg}$, $v_{\text {jet }}=0.17 c, t_{0}=9800 \mathrm{yr}, T_{\text {align }}=7580 \mathrm{yr}, \varphi_{0}=40 \mathrm{deg}$ and $\eta=$ $17 \mathrm{deg}$.

$$
P_{\text {prec }}(t)=P_{0} e^{-\left(t-t_{0}\right) / T_{\text {align }}}
$$

where $\varphi_{0}$ and $P_{0}$ are, respectively, the inclination angle and precession period at time $t_{0}$ when the disk was formed.

To compare our model with observations, we calculated the right ascension and declination offsets $(\Delta \alpha$ and $\Delta \delta$ respectively) of each jet element as a function of time. We assumed that each jet element is receding ballistically from the unresolved core of NGC 1068 with a speed $v_{\text {jet }}$ similarly to [10], 
who analysed the influence of jet precession on the relativistic jet of 3C 120. Unfortunately, only limits to the jet velocity of NGC 1068 are actually known, which ranges approximately from 0.01 to $0.17 c$ (e.g., Ref. 31, 39). This range assures that we can neglected any relativistic correction in our approach (e.g., time-delay effect).

To project the jet motion on the plane of the sky, we introduced two additional parameters: the viewing angle of the jet in relation to the line of sight $\theta$, assumed to be larger than sixty degrees (a very conservative limit for NGC 1068; e.g., Ref. 34), and the position angle of black hole spin axis on the plane of the sky $\eta$. We show in Figure 3 a direct comparison between our model and the parsec-scale jet for $\theta=80$ degrees, $v_{\text {jet }}=0.17 c, t_{0}=9800 \mathrm{yr}, T_{\text {align }}=7580 \mathrm{yr}, \varphi_{0}=40 \mathrm{deg}$ and $\eta=17 \mathrm{deg}$. Our simple kinematic approach reproduces quite well the position of the jet components $\mathrm{C}$ and $\mathrm{NE}$ and the counterjet component S2.

Repeating this procedure to other values of $\theta$ and $v_{\text {jet }}$ constrained by the observations we found that $14 \mathrm{deg} \leq \eta \leq$ $20 \mathrm{deg}, 9600 \leq t_{0}(\mathrm{yr}) \leq 1.7 \times 10^{5}, 7500 \leq T_{\text {align }}(\mathrm{yr}) \leq 1.3 \times$ $10^{5}$ and $25 \mathrm{deg} \leq \varphi_{0} \leq 45 \mathrm{deg}$. The limits for $T_{\text {align }}$ imposed by jet geometry are perfectly compatible with those calculated from Eq. (4).

\section{CONCLUSIONS}

The Bardeen-Petterson effect can be responsible for changes in the accretion disc/jet direction along the time, pro- ducing warps and precession. Such mechanism arises from the frame dragging produced by a Kerr black hole whose its rotation axis is not parallel with that of the accretion disc.

In this work, we explored the consequences of the BardeenPetterson effect to probe the physical characteristics of the accretion disc and the black hole in the nuclear region of the Seyfert 2 galaxy NGC 1068, constraining our analysis to the interferometric radio continuum and maser observations available in the literature.

Using an analytical approach, similar to that suggested by [28] and [23], we calculated the Bardeen-Petterson radius and the alignment time-scale between the accretion disc and the equator plane of the black hole for different values of the black hole spin. We found that the Bardeen-Petterson radius is roughly limited between $10^{-5}$ and $10^{-4} \mathrm{pc}$, while the alignment time-scale ranges from about 100 to $10^{5} \mathrm{yr}$. Those estimates are perfectly compatible with the upper limit to AGN lifetime of NGC $1068\left(\leq 1.5 \times 10^{5} \mathrm{yr}\right)$.

We also showed that the location of each jet component of NGC 1068 is well described by a simple kinematic model, in which jet inlet direction varies with time due to the alignment and precession of the disc. From this approach, we found that the alignment time-scale must be between 7500 and $1.3 \times$ $10^{5}$ years, in order to reproduce correctly the jet component positions.

\section{Acknowledgments}

This work was supported by the Brazilian Agencies FAPESP and CNPq.
[1] N. I. Shakura, R. A. Sunyaev A\&A, 24, 337 (1973).

[2] J. P. De Villiers, J. F. Hawley, and J. H. Krolik ApJ, 599, 1238 (2003).

[3] J. A. Zensus ARA\&A, 35, 607 (1997).

[4] A. C. Gower and J. B. Hutchings ApJ, 258, L63 (1982).

[5] Z. Abraham, E. A. Carrara, J. A. Zensus, and S. C. Unwin A\&AS, 115, 543 (1996).

[6] Z. Abraham and E. A. Carrara ApJ, 496, 172 (1998).

[7] Z. Abraham and G. E. Romero A\&A, 344, 61 (1999).

[8] Z. Abraham A\&A, 355, 915 (2000).

[9] A. Caproni and Z. Abraham ApJ, 602, 625 (2004a).

[10] A. Caproni and Z. Abraham MNRAS, 349, 1218 (2004b).

[11] T. Storchi-Bergmann, R. N. da Silva, and J. P. Halpern, et al. ApJ, 598, 956 (2003).

[12] J. F. Gallimore, S. A. Baum, and C. P. O'Dea ApJ, 613, 794 (2004).

[13] J. I. Katz ApJ, 478, 527 (1997).

[14] G. E. Romero, L. Chajet, Z. Abraham, and J. H. Fan A\&A, 360 $57(2000)$

[15] J. M. Bardeen and J. A. Petterson ApJ, 195, L65 (1975).

[16] D. C. Wilkins PRD, 5, 814 (1972).

[17] A. Caproni, H. J. Mosquera Cuesta, and Z. Abraham ApJ, 616, L99 (2004).

[18] S. Kumar and J. E. Pringle MNRAS, 213, 435 (1985).

[19] P. B. Ivanov and A. F. Illarianov MNRAS, 285, 394 (1997).

[20] R. P. Nelson and J. C. B. Papaloizou MNRAS, 315, 570 (2000).

[21] S. H. Lubow, G. I. Ogilvie, and J. E. Pringle MNRAS, 337, 706 (2002).
[22] P. C. Fragile and P. Anninos ApJ, 623, 347 (2005).

[23] P. Natarajan and P. J. Armitage ApJ, 506, L97 (1999).

[24] P. Natarajan and J. E. Pringle MNRAS, 309, 961 (1998).

[25] G. I. Ogilvie MNRAS, 304, 557 (1999).

[26] J. C. B. Papaloizou and D. N. C. Lin ApJ, 438, 841 (1995).

[27] M. J. Rees Nature, 275, 516 (1978).

[28] P. A. G. Scheuer and R. Feiler MNRAS, 282, 291 (1996).

[29] J. Bland-Hawthorn, J. F. Gallimore, L. J. Tacconi, E. Brinks, S. A. Baum, R. R. J. Antonucci, and G. N. Cecil ApSS, 248, 9 (1997).

[30] W. Jaffe, et al. Nature, 47, 429 (2004).

[31] A. S. Wilson and J. S. Ulvestad ApJ, 319, 105, (1987).

[32] L. J. Greenhill, C. R. Gwinn, R. Antonucci, and R. Barvainis ApJ, 472, L21 (1996).

[33] J. F. Gallimore, S. A. Baum, C. P. O’Dea, and E. Brinks, A. Pedlar ApJ, 462, 740 (1996a).

[34] J. F. Gallimore, C. Henkel, S. A. Baum, I. S. Glass, M. J. Claussen, M. A. Prieto, and A. Von Kap-herr ApJ, 556, 694 (2001).

[35] J.-M. Huré A\&A, 395, L21 (2002).

[36] G. Lodato and G. Bertin A\&A, 398, 517 (2003).

[37] P.J. Sakimoto and F. Coroniti ApJ, 247, 19 (1981).

[38] A. Capetti, D. Axon, F. Macchetto, A. Marconi, and C. Winge, ApJ, 516, 187 (1999).

[39] J. F. Gallimore, S. A. Baum, and C. P. O’Dea ApJ, 464, 198 (1996b). 